\title{
Aportes de los marcadores bioquímicos para el establecimiento de los protocolos en el diagnóstico de sepsis neonatal en el sistema hospitalario docente de la Universidad de Guayaquil
}

\author{
Contributions of biochemical markers for the establishment of protocols in the \\ diagnosis of neonatal sepsis in the teaching hospital system of the University of \\ Guayaquil
}

Contribuições de marcadores bioquímicos para o estabelecimento de protocolos no diagnóstico de sepse neonatal no sistema hospitalar de ensino da Universidade de Guayaquil

\author{
Zoila Luna-Estrella ${ }^{\mathrm{I}}$ \\ zoila.lunae@ug.edu.ec \\ Julio V. Rodríguez-Zurita II \\ julio.rodriguezz@ug.edu.ec \\ Nilda R. Cedeño-Albán III \\ nilda.cedenoa@ug.edu.ec
}

Haydee M. Alvarado-Alvarado IV

haydee.alvaradoa@ug.edu.ec

Laura L. Valdez-López V

laura.valdezl@ug.edu.ec

\footnotetext{
${ }^{\mathrm{I}}$ Docente, Universidad de Guayaquil, Guayaquil, Ecuador.

${ }^{\text {II } D o c e n t e, ~ U n i v e r s i d a d ~ d e ~ G u a y a q u i l, ~ G u a y a q u i l, ~ E c u a d o r . ~}$

III Docente, Universidad de Guayaquil, Guayaquil, Ecuador.

${ }^{\text {IV }}$ Docente, Universidad de Guayaquil, Guayaquil, Ecuador.

${ }^{\mathrm{v}}$ Docente, Universidad de Guayaquil, Guayaquil, Ecuador.
} 


\section{Resumen}

Las enfermedades infecciosas son causas muy importantes de morbilidad y mortalidad en el período neonatal. La Sepsis, es considerada como una de las condiciones que amenazan la mayor parte de este período. La Sepsis es un síndrome clínico que refleja una respuesta inflamatoria sistémica descontrolada ante una infección. Incluye la presencia de un agente infeccioso y al menos algunos de los signos de respuesta inflamatoria sistémica, como alteraciones de la regulación térmica y cambios en la frecuencia cardíaca y respiratoria que suelen ser inicialmente imperceptibles. Cuando la respuesta inflamatoria sistémica se mantiene y agrava el paciente desarrollará disfunción orgánica múltiple, con diferentes grados de shock, seguidos eventualmente por la muerte. La problemática de la aplicación de los marcadores bioquímicos en el laboratorio en la detección de la sepsis neonatal en la unidad de Cuidados Intensivos Neonatal de Centros Hospitalarios, es de gran importancia ante la severa infección en la que los neonatos niños y niñas, están expuestos en las primeras horas de vida, ya que en algunos casos puede llegar a producirse el deceso de dichos pacientes que no está debidamente diagnosticado, ni mucho menos medicado por parte del especialista. El cual no cuenta con un análisis clínico detallado, para poder enfrentar la bacteria que esté ocasionando la sepsis; la investigación realizada de manera bibliográfica, descriptiva, retrospectivo, y transversal, fichas de resultados de laboratorio, de los pacientes neonatos que por distintas causas son asistidos en la unidad de cuidados intensivos del Sistema Hospitalario Docente de la Universidad de Guayaquil, para ello se obtendrán las muestras de sangre de los diferentes pacientes. El objetivo fue describir la metodología empleada para el diagnóstico y control de este evento, se describieron los pasos a seguir ante la sospecha de un brote en estos servicios, pues eleva la morbilidad y mortalidad infantil. Este método permitió la confirmación de los diagnósticos y con ello logren una mejor calidad de vida, utilizando métodos de ensayos bioquímicos y hematológicos, con la ayuda de reactivos y equipos de punta de laboratorio, para obtener resultados específicos con un alto grado de sensibilidad que permita brindar seguridad al momento de diagnosticar por parte del especialista.

La muestra estará conformada por todos los neonatos asistidos en la unidad de cuidados intensivos del hospital, para llevar a cabo este proceso se utilizará una universo conformado por pacientes neonatos de los sexos masculinos y femeninos, de la obtención de estos datos se hará la propuesta de organizar los protocolos de laboratorio de pruebas hematológicas, bacteriológicas, y bioquímicas para maximizar los resultados que ofrezcan una detección rápida y oportuna, en beneficio del neonato que presenta una grave infección intramaterna o extramaterna, así como también infección nosocomial.

Palabras clave: Sepsis neonatal; hospitales pediátricos; mortalidad infantil; marcadores bioquímicos; protocolos; historia clínica. 


\section{Abstract}

Sepsis is a clinical syndrome that reflects an uncontrolled systemic inflammatory response to infection .Include the presence of an infectious agent and at least some of the signs of systemic inflammatory response, such as altered thermoregulation and changes in heart and respiratory rate are usually initially undetectable. When the systemic inflammatory response remains and worsens the patient developed multiple organ dysfunction, with varying degrees of shock, followed eventually by death.

The issue of the application of biochemical markers in the laboratory in the detection of neonatal sepsis in the Neonatal Intensive Care hospital centers, is of great importance to the severe infection in which infants children are exposed to the first hours of life, because in some cases the death of these patients are not properly diagnosed, much less medicated by the specialist can come to occur. Which does not have a detailed, in order to face the bacteria that is causing the sepsis clinical analysis, research on bibliographic, descriptive, retrospective way, and transverse profiles laboratory results of neonatal patients who for various reasons are assisted in the intensive care unit of the Hospital System Professor of the University of Guayaquil, for that blood samples from different patients were obtained. The objective was to describe the methodology used for the diagnosis and management of this event, the steps of a suspected outbreak in these services, as rising infant morbidity and mortality were described. This method allowed the confirmation of the diagnosis and thus achieve a better quality of life, using methods of biochemical and hematological tests, with the help of reagents and laboratory equipment tips to get specific results with a high degree of sensitivity that allows provide security at the time of diagnosis by the specialist . The sample will consist of all infants cared for in the intensive care unit of the hospital, to carry out this process, a universe comprised of neonatal patients of male and female sexes, obtaining these data will be used the proposal will organize lab protocols hematological, bacteriological, and biochemical to maximize results that provide a quick and timely detection, the benefit of the infant who has severe maternal intra or extra- maternal infection, as well as nosocomial infection.

Key words: Neonatal sepsis; pediatric hospitals; mortality biochemical markers; protocols; medical history. 


\section{Introducción.}

Las enfermedades infecciosas son causas importantes de morbilidad y mortalidad en el el período neonatal. Entre ellas, la sepsis es considerada como una de las condiciones que amenazan la mayor parte de este período. La Sepsis Neonatal es un síndrome clínico de infección hematógena con respuesta sistémica a una posible infección, con o sin localización indiferentes sitios del organismo, con hemocultivo positivo o no, que se produce dentro de las primeras cuatro semanas de vida. En relación con el modo de contaminación, se deben diferenciar las sepsis de transmisión vertical, que son causadas por gérmenes localizados en el canal genital materno que contaminan al feto por vía ascendente o por contacto directo del feto con secreciones contaminadas al pasar por el canal del parto; las sepsis nosocomiales, que son debidas a microorganismos localizados en los Servicios de Neonatología (preferentemente en las UCI neonatales) que son transportados al niño por el personal sanitario (manos contaminadas) y/o por el material de diagnóstico y/o tratamiento contaminado, y las sepsis comunitarias, que son debidas a microorganismos que contaminan al RN en su domicilio y que son muy infrecuentes. (Coronell, Pérez, Guerrero, \& Bustamante, 2009) (Briceño, 2005)

El diagnóstico de sospecha de la sepsis neonatal se fundamenta en una serie de factores de riesgo y de parámetros clínicos y analíticos inespecíficos, por lo que en muchas ocasiones resulta difícil valorar cuándo es conveniente iniciar tratamiento antibiótico. (Perotti, Cazales, \& Martell, 2005)

La infección bacteriana es la principal causa de hasta el 30\% de las muertes neonatales y aparece 5 veces más a menudo en los recién nacidos de bajo peso al nacer que en los neonatos a término, ocurre en el 0,5-8,0/1000 recién nacidos vivos; las tasas más elevadas se observan en recién 
nacidos de bajo peso al nacer, los que presentan una función respiratoria deprimida, este riesgo es más pronunciado en los varones y en los recién nacidos con malformaciones congénitas. (Zea-Vera, Turin, \& Ochoa, 2014)

La importancia de esta investigación obedece al riesgo que tienen los niños y niñas al nacer, en el que producto de un proceso de salud en el momento del parto adquieren este tipo de microorganismo, como es el de la sepsis hospitalaria, transmitidos a los niños por el personal sanitario o producida por diferentes causas que han incidido en la morbi-mortalidad de este tipo de pacientes; a decir de los responsables de los centros hospitalarios que se ha logrado llevar determinados programas orientados al cuidado de este tipo de defectos sanitarios, se observa que más bien, no se han venido cumpliendo los manuales de procedimientos y técnicas de limpieza hospitalaria como los ocurridos en hospitales y clínicas públicas y privadas de nuestro país. (De Souza Santos, Costa da Silva, Lima de Souza, \& Miranda Mota, 2014)

Los recién nacidos a término y prematuros tienen mayor riesgo de infección hospitalaria por la inmadurez de su sistema inmunológico y las anomalías congénitas que presenta. Los prematuros tienen un alto riesgo de adquirir cualquier tipo de enfermedad en el proceso de adaptación, debido a que tienen el sistema inmunológico más inmaduro y requieren prolongadas hospitalizaciones y procedimientos invasivos. Los gérmenes que provocan la infección en el periodo neonatal varían según el tipo de sepsis (precoz o tardía), la Epidemiología local de cada hospital y la variación sufrida a través del tiempo. El principal agente causante de la sepsis neonatal es el Estreptococo $\beta$ hemolítico del grupo B (SGB) o Streptococcus agalactiae, que es el germen más frecuente relacionado con sepsis 
neonatales de inicio precoz (50-60\%).Se trata de una bacteria encapsulada cuya virulencia se calcula que entre el 15 y el $25 \%$ de las mujeres embarazadas están colonizadas (genital y anal) por este germen.

Además, otro de los principales agentes etiológicos de sepsis neonatal es la Escherichia Coli, asociada a meningitis neonatal, que se adquiere en el canal del parto y en menor proporción por infección nosocomial.

Muchas bacterias diferentes, incluyendo la, Listeria y ciertas cepas de estreptococo, pueden causar sepsis neonatal.

La Organización Mundial de la Salud (OMS) calcula que en todo el mundo fallecen casi 5 millones de recién nacidos al año y que 98\% ocurren en países en desarrollo. De 30 a $40 \%$ de las muertes neonatales tienen relación con las infecciones. Estrategias implementadas por la OMS, en países de América Latina para disminuir la mortalidad neonatal. La misma fuente consigna que el 90 $\%$ de las muertes neonatales son a consecuencia de hipoxia; problemas congénitos; corta gestación, bajo peso, y sepsis bacteriana. (OMS, 2011)

La mortalidad en todas las edades menores de 5 años es mayor para hijos(as) de madres indígenas que para hijos(as) de mestizas y lo es también para hijos(as) de madres sin instrucción y bajos recursos económicos.

La mortalidad neonatal está más asociada a lo que pasa en el embarazo y parto, que a las condiciones de la vivienda. Aun cuando hay segmentos de la población en los cuales todavía se puede disminuir la mortalidad neonatal mediante la mejoría en las condiciones de vida, es necesario hacer notar que no se puede esperar continuar la disminución de esta mortalidad sólo por estos 
mecanismos. Es necesario el desarrollo y aplicación de estrategias de salud pública a los componentes de mortalidad neonatal, tales como solucionar y prevenir la sepsis neonatal, traumatismo al nacer, anomalías congénitas, y bajo peso y prematuras.

En los medios de la prensa escrita y hablada se dieron informaciones correspondientes al inadecuado manejo de los neonatos y su posterior fallecimiento; lo cual en muchos casos trajo cambios en equipos y personal en hospitales y clínicas, estos niveles infecto contagiosos se produjo por el exceso de polvo en los ductos de los acondicionadores de aire, tomacorrientes oxidados y falta de asepsia, además de haberse diagnosticado la presencia de la bacteria Serratia marscenses en griferías y lavabos.

La Atención Prenatal precoz, se inicia desde el momento en que la madre sospecha la gestación, debiéndose garantizar cuatro atenciones prenatales.

El Ministerio de Salud, para garantizar la atención, pretenden homogenizar el accionar del personal profesional y técnico con el propósito de unificar el proceso de atención y fortalecer la capacidad del personal de los servicios de salud.

La presente investigación tiene como objetivo aplicar los marcadores bioquímicos y hematológicos para la detección oportuna de sepsis neonatal en pacientes asistidos en la Unidad de Cuidados Intensivos Neonatales del Sistema Hospitalario Docente de la Universidad de Guayaquil. 


\section{Metodología.}

El estudio se realizó en la Unidad de Cuidados Intensivos Neonatales del Sistema Hospitalario Docente de la Universidad de Guayaquil. Con la colaboración del laboratorio clínico que es un área destinada a realizar análisis hematológicos, bioquímicos y bacteriológicos del hospital, el cual dispone de la infraestructura y equipos con tecnología de punta necesarios para realizar la investigación, contribuyendo al control de los procesos.

El universo estuvo constituido por 80 pacientes neonatos de ambos sexos, asilados en la Unidad de Cuidados Intensivos Neonatales del Sistema Hospitalario Docente de la Universidad de Guayaquil, durante el período de la investigación

\section{Muestra}

La muestra se conformó con 30 pacientes neonatos con presunción de Sepsis, durante el período de la investigación internados en el área de Cuidados Intensivos Neonatal en el Sistema Hospitalario Docente de la Universidad de Guayaquil.

Para el registro de los datos correspondientes a las variables biológicas, patológicas, socioculturales y conductuales, se diseñó una plantilla (Anexo I ), que fue llenada por el investigador en las entrevistas a los padres de los pacientes.

\section{Tipo de investigación}

Es una investigación Descriptiva y Correlacional. 
Este trabajo se basará en fichas clínicas de los neonatos que son asistidos en la Unidad de Cuidados Intensivos del Sistema Hospitalario Docente de la Universidad de Guayaquil, del análisis de laboratorio específicamente con muestras extraídas de sangre recogida con adecuado cuidado y se obtuvieron los datos necesarios para la investigación. Se confeccionó una base de datos Excel, se emplearon métodos de estadística descriptiva para el análisis de los resultados.

\section{Diseño de investigación}

El diseño de la investigación fue no experimental.

El proceso para el desarrollo de la investigación es el siguiente:

a. Revisión de las Historias .Clínicas, protocolos de tratamiento, estadística de los pacientes diagnosticado con sepsis

b. Toma de muestra

c. Preparar la muestra y procesarla

d. Preparar los equipos.

e. Acondicionar la muestra.

f. Análisis de los resultados.

g. Validación de los resultados

h. Redacción

i. Revisión. 


\section{Técnicas de recolección de datos}

Se estudiaron 80 pacientes de los cuales 30 pacientes neonatos son considerados para el estudio ya que presentaban características de presunción de sepsis neonatal ingresados en la Unidad de Cuidados Intensivos Neonatal ( UCIN )del Sistema Hospitalario Docente de la Universidad de Guayaquil, entre los meses de septiembre 2012- enero 2013, se lo realizó en base al análisis de las Historias Clínicas, para ello se hará uso de la técnica en observación, entrevista, encuesta; los instrumentos de registros de observación, guion de entrevistas, cuestionario, etc.

Para la investigación de marcadores bioquímicos en la detección de sepsis neonatal en pacientes asistidos en la Unidad de Cuidados Intensivos del Sistema Hospitalario Docente de la Universidad de Guayaquil, se realizará la técnica del cuestionario(encuesta) con tres tipos de distractores, tales como:

- SÍ,

- NO,

- A VECES,

Tanto para el segmento pacientes neonatos niños, así como también para el segmento pacientes neonatas niñas del Sistema Hospitalario Docente Universitario de la Universidad de Guayaquil.

Además se realizaron análisis de laboratorio:

Hematológicos como Hemoglobina, (g/dl), Hematocrito ( \% ), Grupo Sanguíneo, VCM, Glóbulos Rojos, Glóbulos Blancos, Velocidad de Sedimentación Globular, Plaquetas, T.P, TPT.

Linfocitos, Neutrófilos, Monocitos, etc. 
Bioquímicos como Glicemia, Urea, Creatinina, Ácido Úrico, PCR, Procalcitonina, TGO, TGP, BT, BD. BI, Gasometría, Sodio, Potasio, Cultivo, Hemocultivo, Anti-Toxoplasma, Sangre Oculta, de los pacientes en estudio y establecer el diagnóstico dado por el médico de los paciente y diferenciar de los que si presentaron Sepsis.

- Los pacientes Neonatos se los calificó en base a los formularios empleados por el SHDUG y dados por el Ministerio de Salud Pública que contienen:

- Historia Clínica del Recién Nacido (datos del recién nacido, tipo de parto, hora y fecha de nacimiento, sexo, causa del parto, frecuencia cardiaca, frecuencia respiratoria, etc. ).

- Datos de la madre (nombre, C.I., HC, edad, ocupación, dirección, control del embarazo, grupo sanguíneo, etc ).

- Datos del padre (nombre, edad, C.I, ocupación, dirección, grupo sanguíneo,etc ).

\section{Resultados.}

Según la Organización Mundial de la Salud, hasta el año 2012, las estadísticas indicaron que el $27 \%$ de muertes Neonatales fueron producidas por Sepsis y Neumonías, siendo el $25 \%$ por Asfixia y un $16 \%$ por malformaciones congénitas.

En el período de estudio entre septiembre 2012 a enero del 2013 en el área de pediatría las madres de los neonatos que tenían factores de riesgo de sepsiseran el $60 \%$ de origen urbano y el 40 $\%$ de origen rural, siendo éste último grupo el más susceptible por tener poco acceso a los servicios de salud, menos control prenatal y condiciones socioeconómicas desfavorables.

En el período de estudio entre septiembre 2012 a enero del 2013 en el área de pediatría las madres de los neonatos en estudio, presentaban las siguientes edades : 18 madres sus edades 
llegaban a 20 años, fluctuando desde los 16 años, 9 tenían más de 20 años llegando a 25 y 1 era mayor de 35 años, observándose en el estudio que las primeras eran el grupo más susceptible por tener poca responsabilidad emocional, baja autoestima y poco nivel de educación, descuido en buscar los servicios de salud, menos control prenatal y condiciones socioeconómicas desfavorables.

En el período de estudio de septiembre 2012 a enero del 2013, de las madres de los 30 casos de neonatos en estudio, el $18 \%$ poseían educación inicial, sabiendo leer y escribir. El 7 \% no poseía ninguna educación y apenas el $5 \%$ poseía educación primaria completa y secundaria incompleta.

En el período de estudio de septiembre 2012 a enero del 2013, de los 30 neonatos estudiados, 19 eran de sexo masculino y 11 de sexo femenino.

En la presente tabla con respecto a la edad de los neonatos concluimos que el mayor porcentaje de los pacientes evaluados corresponde al grupo de menor a las 72 horas. De vida. Determina horas de vida, refleja transmisión horizontal de la comunidad o intra hospitalaria, argumentando que el sexo masculino tiene un riesgo mayor veces mayor que el sexo femenino.

En el período de estudio de septiembre 2012 a enero del 2013, observamos la frecuencia del tipo de parto y de embarazo en los neonatos estudiados, 28 partos fueron por cesárea y dos fueron parto Normal. Observándose el predominio de cesárea por las condiciones en que asistían las madres a la Casa Asistencial. Las indicaciones de : la cesárea son realizarlas cuando el parto vaginal puede ser arriesgado para el niño o la madre, embarazo múltiple, evidencia de infección intrauterina y sufrimiento fetal aparente

En el período de estudio de septiembre 2012 a enero del 2013, de los 30 neonatos estudiados, 
13 neonatos presentaron cuadro clínico de sepsis del recién nacido y 17 neonatos presentaron diferentes enfermedades.

En el período de estudio de septiembre 2012 a enero del 2013, de los 30 neonatos estudiados con presunción de sepsis neonatal, 17 neonatos fueron transferidos de otros Hospitales y 13neonatos fueron pacientes que llegaron al SHDUG, para su diagnóstico.

En el período de estudio de septiembre 2012 a enero del 2013, de los 30 neonatos estudiados con presunción de sepsis neonatal, provenientes de otros hospitales urbanos y rurales, incluidos los del SHDUG, 25 presentaron Mejoría Clínica y 5 Fallecenpor Sepsis Bacteriana del Recién Nacido.

En el período de estudio de septiembre 2012 a enero del 2013, de los 30 neonatos estudiados con presunción de sepsis neonatal, presentaron los siguientes pesos, encontrándose fuera de los valores normales todos los pacientes estudiados.

En el período de estudio de septiembre 2012 a enero del 2013, de los 30 neonatos estudiados con presunción de sepsis neonatal, dentro de una de las pruebas realizadas de laboratorio, el Hemocultivo, se observó que arrojó de los 30 pacientes, 10 con resultados Negativos, a 9 no se les envío la prueba y el restante presentaron resultados positivos pero en muy poco porcentaje.

Analizamos un parámetro del score, el contaje total de leucocitos en los neonatos, dando como resultado la presencia de alteraciones en el contaje de leucocitos. El recuento leucocitario es un estudio indirecto valioso para la determinación de sepsis neonatal definiendo que la leucocitosis y la leucopenia son determinantes en una infección.

En esta tabla observamos un parámetro del score, el contaje total de plaquetas dando como resultado la no presencia de alteraciones de las plaquetas en 24 pacientes, y menores a $150.000 / \mathrm{m}^{3}$ 
de plaquetas en 6 pacientes, de los cuales 5 fallecen posteriormente. Observando que solo un pequeño grupo tiene anormalidades en el contaje total de plaquetas Siendo un problema hematológico común en las unidades de terapia intensiva y que juega un papel importante en el pronóstico de los pacientes críticos. Su importancia radica que constituye un marcador de riesgo rápido y sencillo de evaluar para valorar la mortalidad y el pronóstico de los pacientes durante la evolución del proceso séptico.

En el período de estudio de septiembre 2012 a enero del 2013, de los 30 neonatos estudiados con presunción de sepsis neonatal, dentro de una de las pruebas realizadas de laboratorio PCR (es una proteína de fase aguda que aumenta en el suero resultado de la liberación de interleuquina 6. Indica la presencia de inflamación) se observó que arrojó de los 30 pacientes, 3 con resultados Negativos, a 6 no se les envío la prueba y el restante 21, presentaron resultados positivos, observándose procesos inflamatorios en los pacientes neonatos de estudio.

\section{Conclusiones.}

Existen fuertes fundamentos teóricos que sustentan la necesidad del desarrollo de un protocolo de acción, para contribuir a la disminución de la tasa de morbilidad y mortalidad por sepsis neonatal precoz.

Se observó que las condiciones de desnutrición de las embarazadas que condicionan partos prematuros, malformaciones congénitas y bajo peso al nacer.

La falta de Medicamentos e Insumos Médicos, contribuían a la mala atención.

Que la falta de capacitación del personal médico nuevo o con poca experiencia en la atención de embarazos de riesgo, lo que elevaba las probabilidades de muerte del 
neonato por hipoxia y asfixia al nacer.

Un bajo nivel cultural y educativo de la embarazada y su familia, que hacen que ésta no recurra a los servicios Institucionales. Las Limitaciones económicas de la familia que asociadas a dificultades de acceso geográfico limitan el acceso a los servicios institucionales.

La Baja efectividad de la atención primaria de la salud, lo que se refleja en censos nominales no actualizados; seguimiento inoportuno del embarazo y detección tardía del embarazo de alto riesgo.

Rechazo de la embarazada para la atención del parto.

Elevada atención de partos por parteras tradicionales muchas veces no supervisadas por personal médico.

El desamor de las embarazadas muy jóvenes al producto que se encuentra en su vientre y al desamor y descuido del personal médico, enfermeras, no ayudan a una buena atención.

Las manipulaciones previas al ingreso en el hospital, el parto inducido / conducido, el número de tactos, la mayor duración del parto y las maniobras invasivas son claros factores de riesgo.

Los partos durante la noche tienen más riesgo de sepsis, lo que podría evidenciar algún problema organizativo.

Toda la información obtenida se la procesó en una base de datos, confeccionada en un sistema de computación compatible con el análisis estadístico básico, donde se emplearon los sistemas Microsoft Word, Excel, Power Point. 
La información se la presentó en tablas de contingencia, elaboradas en Excel, utilizando la base de datos y facilitar el análisis y presentación de los resultados obtenidos.

\section{Bibliografía.}

Briceño, I. (2005). Sepsis: Definiciones y aspectos fisiopatológicos. Medicrit, 2(8), 164-178.

Coronell, W., Pérez, C., Guerrero, C., \& Bustamante, H. (2009). Sepsis neonatal. Revista de Enfermedades Infecciosas en Pediatría, 23(90), 57-68.

De Souza Santos, A., Costa da Silva, M., Lima de Souza, N., \& Miranda Mota, G. (2014). Diagnósticos de enfermería de recién nacidos con sepsis en una Unidad de Terapia Intensiva Neonatal. Rev. Latino-Am. Enfermagem, 22(2), 255-261.

OMS. (2011). Disminuye la mortalidad neonatal, pero aumenta su proporción en la mortalidad en la niñez a escala mundial. Ginebra: Organización Mundial de la Salud.

Perotti, E., Cazales, C., \& Martell, M. (2005). Estrategias para el diagnóstico de sepsis neonatal tardía. Revista Médica del Uruguay, 21(4), 314-320.

Zea-Vera, A., Turin, C., \& Ochoa, T. (2014). Unificando los criterios de sepsis neonatal tardía: propuesta de un algoritmo de vigilancia diagnóstica. Rev Peru Med Exp Salud Publica, $31(2), 358-363$. 